\title{
Early Miocene origin and cryptic diversification of South American salamanders
}

\author{
Kathryn R Elmer ${ }^{1,2,3^{*}}$, Ronald M Bonett ${ }^{4,5}$, David B Wake ${ }^{5^{*}}$ and Stephen C Lougheed ${ }^{1}$
}

\begin{abstract}
Background: The currently recognized species richness of South American salamanders is surprisingly low compared to North and Central America. In part, this low richness may be due to the salamanders being a recent arrival to South America. Additionally, the number of South American salamander species may be underestimated because of cryptic diversity. The aims of our present study were to infer evolutionary relationships, lineage diversity, and timing of divergence of the South American Bolitoglossa using mitochondrial and nuclear sequence data from specimens primarily from localities in the Andes and upper Amazon Basin. We also estimated time of colonization of South America to test whether it is consistent with arrival via the Panamanian Isthmus, or land bridge connection, at its traditionally assumed age of 3 million years.
\end{abstract}

Results: Divergence time estimates suggest that Bolitoglossa arrived in South America from Central America by at least the Early Miocene, ca. 23.6 MYA (95\% HPD 15.9-30.3 MYA), and subsequently diversified. South American salamanders of the genus Bolitoglossa show strong phylogeographic structure at fine geographic scales and deep divergences at the mitochondrial gene cytochrome $b(C y t b)$ and high diversity at the nuclear recombination activating gene-1 (Rag1). Species often contain multiple genetically divergent lineages that are occasionally geographically overlapping. Single specimens from two southeastern localities in Ecuador are sister to the equatoriana-peruviana clade and genetically distinct from all other species investigated to date. Another single exemplar from the Andes of northwestern Ecuador is highly divergent from all other specimens and is sister to all newly studied samples. Nevertheless, all sampled species of South American Bolitoglossa are members of a single clade that is one of several constituting the subgenus Eladinea, one of seven subgenera in this large genus.

Conclusions: The ancestors of South American salamanders likely arrived at least by the Early Miocene, well before the completion of the Late Pliocene Panamanian land bridge (widely accepted as ca. 3 MYA). This date is in agreement with recent, controversial, arguments that an older, perhaps short-lived, land connection may have existed between South America and present-day Panama 23-25 MYA. Since its arrival in South America, Bolitoglossa has diversified more extensively than previously presumed and currently includes several cryptic species within a relatively small geographic area. Rather than two upper Amazonian species currently recorded for this region, we propose that at least eight should be recognized, although these additional lineages remain to be formally described.

\footnotetext{
*Correspondence: kathryn.elmer@glasgow.ac.uk; wakelab@berkeley.edu 'Department of Biology, Queen's University, Kingston, Ontario, Canada ${ }^{2}$ Lehrstuhl für Zoologie und Evolutionsbiologie, Department of Biology, University of Konstanz, Universitätstrasse 10, Konstanz 78457, Germany Full list of author information is available at the end of the article
}

\section{Biomed Central}

(c) 2013 Elmer et al.; licensee BioMed Central Ltd. This is an Open Access article distributed under the terms of the Creative Commons Attribution License (http://creativecommons.org/licenses/by/2.0), which permits unrestricted use, distribution, and reproduction in any medium, provided the original work is properly cited. 


\section{Background}

While the forests of South America are renowned for their diversity of frogs, including endemic clades that are both old and species rich [1-11], South American salamanders are represented only by the family Plethodontidae [12] and are considered species poor in this region. In fact, with the exception of two species of Oedipina, all salamanders in South America belong to the genus Bolitoglossa (subgenus Eladinea) with only 28 currently recognized species that are distributed across the entire continent [12]. Species richness is greatest in Colombia and rapidly decreases to the south and east. Less than one-ninth of all recognized neotropical salamander species are found in South America, despite its geographical area dwarfing that of Central America where the remaining diversity is concentrated [13].

One hypothesis for the lower species richness of South American salamanders is that they arrived only relatively recently via the Isthmus of Panama (or Panamanian land bridge), which is widely accepted to have closed 3 to 4 million years ago (MYA). If true, then South American plethodontids would have had less time to diversify compared to those of Central America (discussed in [13,14] and references therein; tested in [15]). However, recent geological research suggests that the Panamanian land bridge may be much older (ca. 23-25 MYA, [16,17] and references therein), although this remains controversial (Coates, personal communication to DBW, 8 Nov. 2011)

When, and how many times, salamanders migrated into South America has been a matter of debate: for example, Brame \& Wake [18] suggested multiple migrations from the Pliocene to the Pleistocene, while Dunn [19] suggested a Late Miocene to Early Pliocene origin that would predate a $3 \mathrm{MY}$ old land bridge. Estimates based on allozyme distances suggested the divergence between Central and South American species to be at least 18 MYA [20], while analyses from mitochondrial (mt) DNA sequences suggested Bolitogloss $a$ were present in South America before the Pliocene land bridge closure $[13,21]$. Hanken \& Wake [20] offered two alternative hypotheses to explain such a deep level of divergence: 1 ) that several deeply divergent lineages occurred in southern Central America by the Early Pliocene and each independently migrated to South America after the Late Pliocene connection was established, or 2) that Bolitoglossa colonized South America prior to the late Pliocene land bridge and subsequently diversified in situ. Parra-Olea, García-París \& Wake analysed the phylogenetic relationships across Bolitoglossa and concluded that the South American lineages must be old (35.8-12.5 MYA, depending on the molecular clock) and predate the Isthmus of Panama [13]. This was reaffirmed with more advanced chronogram analysis, which calculated a divergence of at least 11 million years [21]. However, to date, there have been no detailed studies or discussion of South American plethodontids based on DNA sequence data and these hypotheses have never been rigorously tested.

Very little is known about South American salamanders because of the "fractal" [22], highly spatially partitioned nature of plethodontid diversity across vast underexplored areas of the upper Amazonian forests. In particular the systematics of Amazonian salamanders has proven difficult because they are generally small, have similar derived morphological features such as extensively webbed digits, reduced dentition, and subtle colour pattern differentiation [23]. Further, they have been seldom collected and thus we are missing even basic data on geographical distributions. In 1963, Brame \& Wake noted that because "... biologists have always been intrigued with organisms living under novel or unusual conditions, or in unexpected regions ... it is surprising that South American salamanders virtually have been ignored for so long" ([18] p. 5). Almost fifty years after this statement, there has been little progress towards quantifying the biodiversity and describing the evolutionary relationships of South American salamanders.

The aim of our present study is two-fold. First, we use divergence time estimates and ancestral area reconstruction to estimate the deepest age of an endemic South American clade and thereby infer the minimum time of colonization of South America by plethodontid salamanders. Our estimates of divergence times suggest that the salamanders arrived and diversified within South America in the Early Miocene. Furthermore, South American Bolitoglossa are all members of a single clade that is nested within a larger clade, the subgenus Eladinea, which also occurs in Costa Rica and Panama. Therefore, their colonization of South America significantly predates the widely accepted date of about 3 MYA for the Panamanian land bridge connection. Because these salamanders have low vagility and most likely reached South America by land, our results are more compatible with a much earlier land bridge connection (e.g. $[16,17])$. Second, we infer evolutionary relationships and the extent of genetic divergence among South American Bolitoglossa from the Ecuadorean Andes and upper Amazon basin (Figure 1) using mitochondrial and nuclear DNA sequence data. Our analyses reveal previously unsuspected deep genetic divergences even within local regions, documenting the presence of extensive cryptic species richness.

\section{Results and discussion}

\section{Bolitoglossa colonization of South America}

Ancestral area reconstruction based on 56 bolitoglossine taxa strongly supported a Central American origin of Bolitoglossa (Figure 2). Our estimate for the deepest time of divergence for Bolitoglossa from throughout Central 


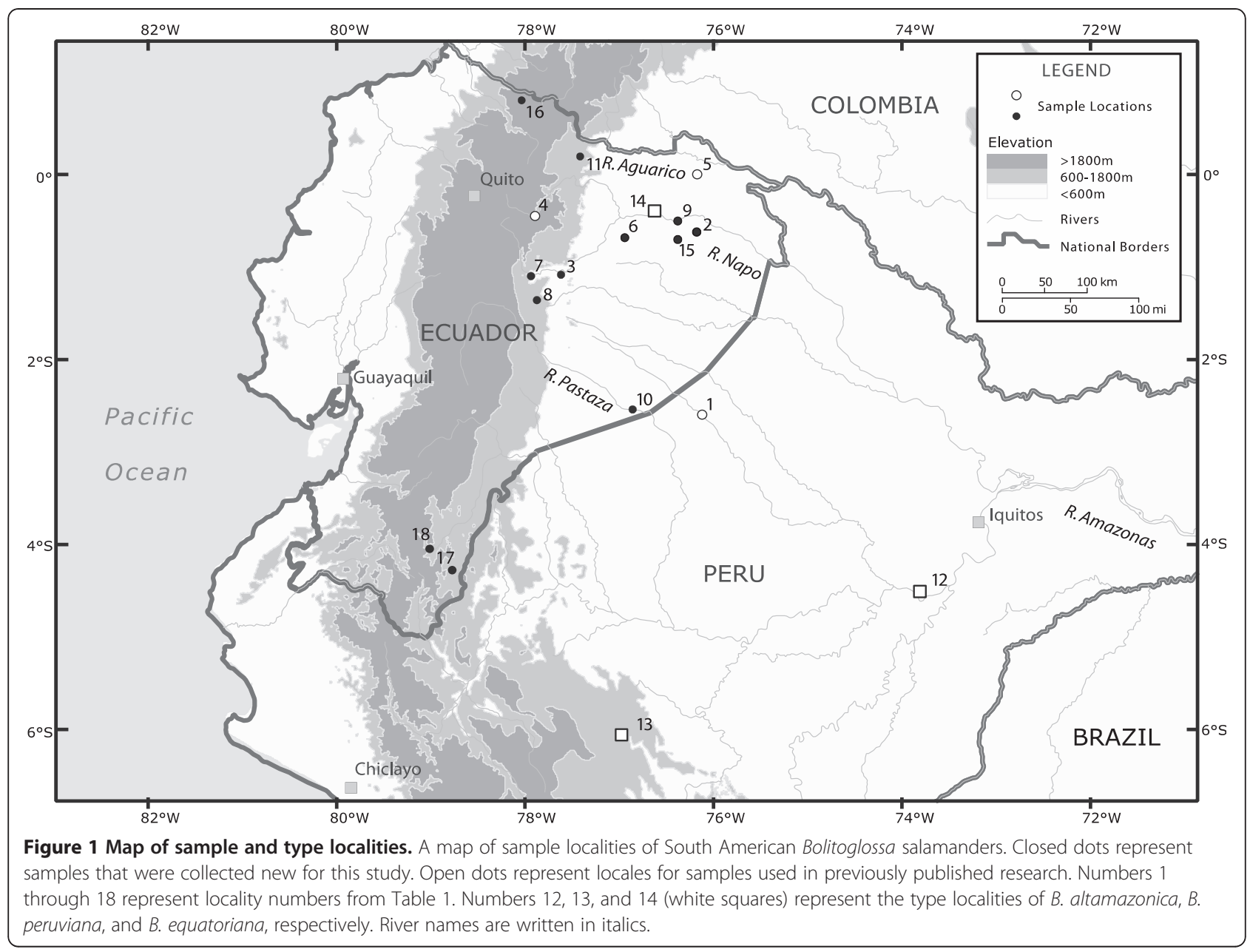

and South America is ca. 50 MYA (95\% highest posterior density [HPD] 36.4 to 62.8 MYA, mean 50.3 MYA), and our estimate for the subgenus Eladinea is ca. 36 MYA (95\% HPD 26.8 to 46.7 MYA, mean 36.5 MYA) (Figure 2, Additional file 1, Additional file 2).

While we were unable to sample the South American taxa exhaustively, we found that the diverse clade of Bolitoglossa from the Andes and Amazon had a high likelihood of a South American origin. The origin of the entire South American clade of sampled Bolitoglossa was estimated to be ca. 23 MYA (95\% HPD 15.9 to 30.3 MYA, mean 23.6 MYA; Figure 2). Even if we consider a much younger divergence time calibrations for the Plethodontidae (60 MYA), we still estimate divergence among sampled South American taxa to be at least 12.7 MYA (Additional file 2). Furthermore, the deep divergence among regionally structured clades within upper Amazonian Bolitoglossa - B. peruviana (ca. 11 MYA [95\% HPD 6.9 to 15.4 MYA, mean 10.8 MYA]) and $B$. equatoriana (ca. 7 MYA [95\% HPD 0.5 to 15.1 MYA, mean 7.4 MYA]) - also confirmed the minimally Miocene age of this radiation within South America
(Figure 2, Figure 3, Additional file 1, Additional file 2). Based on these observations, we reason that the Andean and Amazonian species did not diversify north of the Panamanian land bridge and subsequently disperse into South America after its closure (one of the possibilities advanced by Hanken \& Wake [20]). Rather, the lineages result from diversification in South America after colonization ca. 23 MYA. This is in agreement with the scenario proposed by Parra-Olea et al. [13], whereby the South American Bolitoglossa greatly predate a 3 MYA land bridge between Central and South America.

Until very recently it had generally been accepted that the South American continent was isolated from the northern land mass until the Isthmus permanently closed ca. 3 [24] to 4 [25] MYA. However, recent geological analyses, which are still controversial, suggest the Isthmus may have closed at least for some period of time much earlier. This involved a major narrowing of the seaway and great shifting of islands in the Early Miocene (ca. 23-25 MYA) $[16,17]$ and a complete closure is now estimated to have happened by 15 MYA [26]. Clearly this would have facilitated dispersal between Central and South America. 


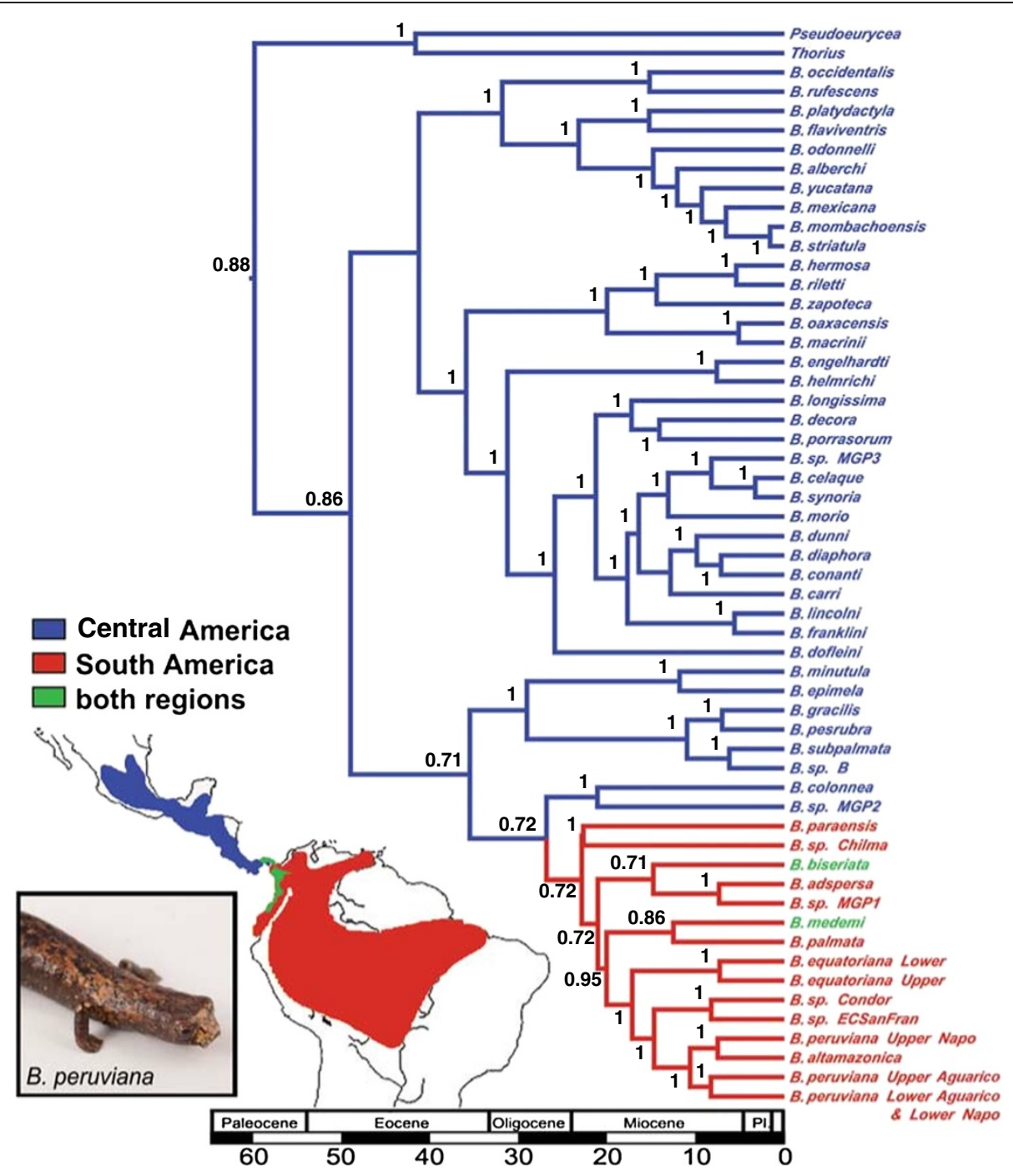

Figure 2 Chronogram and ancestral area reconstruction for Central and South American Bolitoglossa based on Rag1 and Cytb. Mean divergence times are based on Bayesian species tree estimation (non-bolitoglossines are not shown). Biogeographic reconstruction was calculated in Lagrange. The highest split probability for the ancestral area subtends each node, and branches are colored accordingly: Central America (blue), South America (red), and both regions (green). These analyses show a single origin of South American Bolitoglossa by the Early Miocene, and two recent secondary re-colonizations of Central America by species that occur in both regions.

While the Pliocene 'Great American Interchange' between continents approximately 3 MYA considerably affected mammalian fauna [25] and some avian fauna [27,28], a growing list of biological data based on molecular phylogenetics also supports an earlier, Miocene, land connection: e.g. frogs [29,30], plants [31], procyonid mammals [32], and snakes [33]. Our finding confirms this also to be true for plethodontid salamanders. Our estimates of divergence among South American and even just Andean Bolitoglossa are far older than the most generally accepted dates for completion of the Panamanian land bridge $c a$. 3 MYA and instead suggest a very Early Miocene colonization of South America, which is concordant with the postulated land connection between Panama and
South America on the order of 23 to 25 MYA, timing that has been advanced to explain new geological discoveries $[16,17]$.

Since that time there have been at least two other dispersals across the Isthmus by salamanders. The two species in our analyses that occur in Colombia and Panama (B. biseriata and B. medemi) have clear South American origins but the specimens are from Central America and likely represent a more recent secondary colonization of Central America (Figure 2). Other than Bolitoglossa, Oedipina salamanders are also found in South America and these are less well known. The current taxonomy, dating from 1963 [18], recognizes two species, both of which occur in Panama. Of these, O. complex (type 


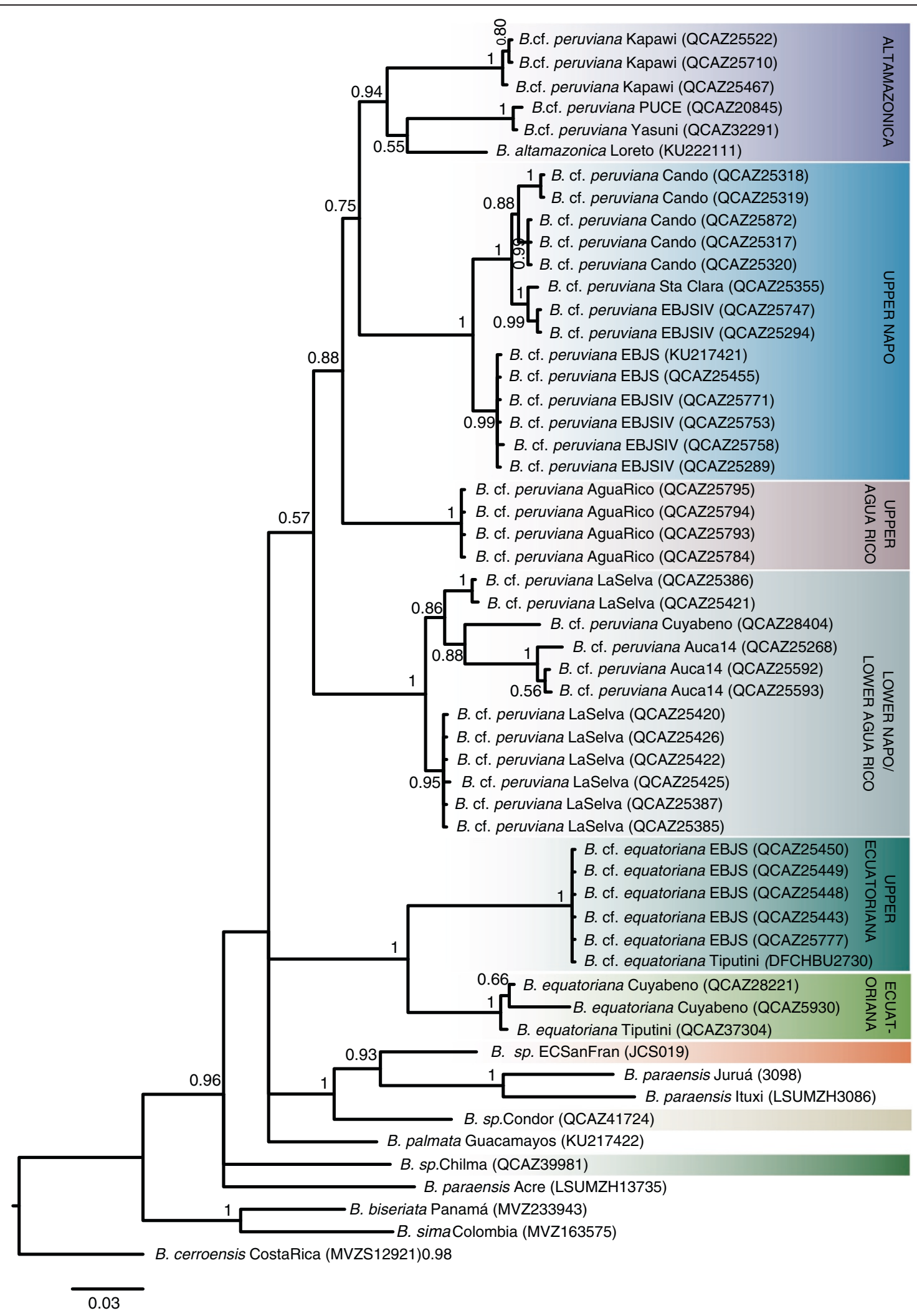

Figure 3 MtDNA phylogeny focusing on South American salamanders. The 50\% majority-rule phylogenetic tree of Cytb inferred from Bayesian analyses. Posterior probabilities are located at nodes or above branches. Subclades referred to in the text and derived from the combined analysis are noted in vertical. See Table 1 and Figure 1 for more information on sample localities. 
locality Gamboa, Panama) is known from two South American specimens, one from Isla Gorgona, Colombia, and the other from northwestern Ecuador. The Ecuadorean specimen has recently been re-examined (DBW, 2011) and represents an unnamed species. The second species, O. parvipes (type locality Cáceras, Antioquia, Colombia), is currently thought to occur in Panama, but it, too, requires a modern taxonomic revision and it is unlikely that the Panamanian and Colombian samples represent the same species. Nevertheless, whether the taxonomy is correct or not, Oedipina is represented by few species and in South America is found only in western Colombia and northern Ecuador. Pending study of currently unavailable samples using molecular methods, Oedipina may well represent a post-isthmus dispersal from its Central American origin [34].

\section{Phylogenetic and phylogeographic patterns within South American Bolitoglossa}

Our phylogenetic analyses based on mtDNA document high diversity of Bolitoglossa lineages in the upper Amazon and Andes of Ecuador, even within a small geographic area (Figure 1, Figure 3). Additionally, there is striking geographical structure in B. equatoriana and B. peruviana often coincident with east Andean and upper Amazonian geographical features. For example, sister clades generally differ in elevation (upland vs. lowland) within and between river basins.

\section{Bolitoglossa equatoriana (sensu lato) vs. B. equatoriana (sensu stricto)}

Bolitoglossa equatoriana (sensu lato) forms a wellsupported monophyletic group (posterior probability [pp] $=1$ ) with two divergent subclades that are separated by elevation and found in the Napo and Aguarico River Basins (Figure 3). One of these we refer to as "upper equatoriana" because it includes specimens from an upland locality on the Napo River (map locality 3; 400 metres above sea level [masl]) and a single specimen from the more lowland Tiputini locality (map locality 2; 250 masl) (Table 1, Figure 4). Its sister subclade includes samples from localities near to, and more geographically typical of, the type locality [35] (map locality 14; 260 masl) and so we consider this group to represent $B$. equatoriana (sensu stricto).

Average genetic divergence between these two subclades of B. equatoriana (sensu lato) is $11.7 \%$ for Cytb (Table 2) and $0.8 \%$ for Rag1 (Table 3). Bayesian estimates of divergence times average ca. 7.4 MYA (Figure 2).

\section{Bolitoglossa peruviana diversity}

Within B. peruviana (sensu lato) four major subclades are broadly distributed by elevation and river basin (Figure 3,
Figure 4). We informally identify these subclades as follows: altamazonica, Upper Napo, Upper Aguarico, and Lower Napo/Lower Aguarico. The altamazonica subclade spans the greatest latitude: from the lower Napo to the lower Pastaza River Basins (map localities 1, 10, 15). This subclade, which includes the most lowland representative in our study (B. altamazonica Loreto), is not the sister taxon of the other Amazonian basin lowland species from Brazil, tentatively assigned to $B$. paraensis. Instead, the specimens assigned to $B$. paraensis (from Ituxi and Juruá) are more closely related to the southeastern Ecuadorean specimens from higher elevations (localities 17 and 18). The upper Napo subclade occurs in multiple localities in the vicinity of the headwaters of the Napo River (localities 3,7 and 8). Although the southeastward distribution of this subclade remains to be determined, it is replaced to the north at the headwaters of the Aguarico River (locality 11; 610 masl) with the divergent sister subclade upper Aguarico. Specimens in the subclade lower Napo/lower Aguarico were found to span the lower Napo and lower Aguarico river basins (localities 5, 6, 9). Additional sampling is required to determine the eastward distribution of this subclade.

The four major subclades of B. peruviana (sensu lato) are highly divergent: from 9.5 to $11.9 \%$ at Cytb (Table 2) and from 1.1 to $2.8 \%$ at Rag1 (Table 3; see Additional files 3 and 4 for inter-individual distances). Divergence time estimates among populations that have been assigned to $B$. peruviana average $c a .10 .8$ MYA (Figure 2).

Given the highly localized nature of genetic differentiation in what has been considered to be B. peruviana, we think that none of the Ecuadorean samples should be assigned to that taxon. Instead, B. peruviana should be considered to be a Peruvian endemic that so far is known only from the unique holotype (locality 12 in Figure 1). Further sampling, especially in this area of southern Ecuador and northern Peru, is required to further resolve phylogenetic relationships.

Our mtDNA phylogeny contains more extensive sampling of upper Amazonian salamanders than the ParraOlea et al. [13] mtDNA phylogeny, which contained samples across the large, geographically widespread genus Bolitoglossa. Parra-Olea et al. [13] considered their specimen from Cuyabeno (locality 5 ) to be $B$. peruviana (QCAZ05930, was LSUMZ-H12838 in [13]). We find this specimen instead to be a member of the $B$. equatoriana (sensu stricto) clade and was likely previously misidentified. We do not have a Rag1 sequence for this specimen.

\section{Ecuadorean highland Bolitoglossa spp}

Taking a broader geographical perspective on our mtDNA phylogenetic hypothesis, the high Andean 
Table 1 Sample information

\begin{tabular}{|c|c|c|c|c|c|c|c|c|c|}
\hline Species & & $\begin{array}{l}\text { mtDNA } \\
\text { Clade }\end{array}$ & $\begin{array}{l}\text { Specimen } \\
\text { number }\end{array}$ & Country & $\begin{array}{l}\text { Province/ } \\
\text { Territory }\end{array}$ & Locality & $\begin{array}{c}\text { Map } \\
\text { Locality } \\
\text { Number }\end{array}$ & cytb accession & $\begin{array}{c}\text { Rag1 } \\
\text { accession }\end{array}$ \\
\hline Bolitoglossa & altamazonica & altamazonica & KU222111 & Peru & Loreto & $\begin{array}{l}1.5 \text { km N Teniente } \\
\text { López }\end{array}$ & 1 & AY526160 & \\
\hline Bolitoglossa & biseriata & outgroup & MVZ232943 & Panama & Kuna Yala & Nusagandi & - & AY526161 & KC614436 \\
\hline Bolitoglossa & cerroensis & outgroup & MVZ233516 & $\begin{array}{l}\text { Costa } \\
\text { Rica }\end{array}$ & Cartago & Prov. Cartago & - & - & KC614459 \\
\hline Bolitoglossa & cerroensis & outgroup & MVZS12921 & $\begin{array}{l}\text { Costa } \\
\text { Rica }\end{array}$ & San José & $\begin{array}{l}\text { Cuericí } 5 \text { km E Villa } \\
\text { Mills }\end{array}$ & - & AF199195 & - \\
\hline Bolitoglossa & $\begin{array}{l}\text { cf. } \\
\text { equatoriana }\end{array}$ & $\begin{array}{c}\text { upper } \\
\text { equatoriana }\end{array}$ & $\begin{array}{l}\text { DFCH- } \\
\text { BU2730 }\end{array}$ & Ecuador & Napo & $\begin{array}{l}\text { Estación Biológica } \\
\text { Jatun Sacha (EBJS) }\end{array}$ & 3 & DQ353846 & \\
\hline Bolitoglossa & $\begin{array}{l}\text { cf. } \\
\text { equatoriana }\end{array}$ & $\begin{array}{c}\text { upper } \\
\text { equatoriana }\end{array}$ & QCAZ25443 & Ecuador & Napo & $\begin{array}{l}\text { Estación Biológica } \\
\text { Jatun Sacha (EBJS) }\end{array}$ & 3 & DQ353841 & \\
\hline Bolitoglossa & $\begin{array}{l}\text { cf. } \\
\text { equatoriana }\end{array}$ & $\begin{array}{c}\text { upper } \\
\text { equatoriana }\end{array}$ & QCAZ25448 & Ecuador & Napo & $\begin{array}{l}\text { Estación Biológica } \\
\text { Jatun Sacha (EBJS) }\end{array}$ & 3 & DQ353842 & KC614451 \\
\hline Bolitoglossa & $\begin{array}{l}\text { cf. } \\
\text { equatoriana }\end{array}$ & $\begin{array}{c}\text { upper } \\
\text { equatoriana }\end{array}$ & QCAZ25449 & Ecuador & Napo & $\begin{array}{l}\text { Estación Biológica } \\
\text { Jatun Sacha (EBJS) }\end{array}$ & 3 & DQ353843 & \\
\hline Bolitoglossa & $\begin{array}{l}\text { cf. } \\
\text { equatoriana }\end{array}$ & $\begin{array}{c}\text { upper } \\
\text { equatoriana }\end{array}$ & QCAZ25450 & Ecuador & Napo & $\begin{array}{l}\text { Estación Biológica } \\
\text { Jatun Sacha (EBJS) }\end{array}$ & 3 & DQ353844 & \\
\hline Bolitoglossa & $\begin{array}{l}\text { cf. } \\
\text { equatoriana }\end{array}$ & $\begin{array}{c}\text { upper } \\
\text { equatoriana }\end{array}$ & QCAZ25777 & Ecuador & Napo & $\begin{array}{l}\text { Estación Biológica } \\
\text { Jatun Sacha (EBJS- } \\
\text { Inner Vision Lodge) }\end{array}$ & 3 & DQ353840 & \\
\hline Bolitoglossa & cf. peruviana & altamazonica & QCAZ20845 & Ecuador & Orellana & $\begin{array}{l}\text { Estación Científica } \\
\text { Yasuní (PUCE) }\end{array}$ & 15 & KC614427 & KC614453 \\
\hline Bolitoglossa & cf. peruviana & altamazonica & QCAZ25467 & Ecuador & Pastaza & Kapawi Lodge & 10 & DQ353811 & \\
\hline Bolitoglossa & cf. peruviana & altamazonica & QCAZ25522 & Ecuador & Pastaza & Kapawi Lodge & 10 & DQ353809 & KC614442 \\
\hline Bolitoglossa & cf. peruviana & altamazonica & QCAZ25710 & Ecuador & Pastaza & Kapawi Lodge & 10 & DQ353810 & \\
\hline Bolitoglossa & cf. peruviana & altamazonica & QCAZ32291 & Ecuador & Orellana & $\begin{array}{l}\text { Estación Científica } \\
\text { Yasuní (PUCE) }\end{array}$ & 15 & KC614430 & KC614455 \\
\hline Bolitoglossa & cf. peruviana & $\begin{array}{l}\text { lower Napo/ } \\
\text { lower } \\
\text { Aguarico }\end{array}$ & QCAZ25268 & Ecuador & Orellana & $\begin{array}{l}\text { Auca } 14 \text { Rd near } \\
\text { Coca }\end{array}$ & 6 & DQ353830 & KC614447 \\
\hline Bolitoglossa & cf. peruviana & $\begin{array}{l}\text { lower Napo/ } \\
\text { lower } \\
\text { Aguarico }\end{array}$ & QCAZ25385 & Ecuador & Orellana & La Selva Lodge & 9 & DQ353835 & KC614449 \\
\hline Bolitoglossa & cf. peruviana & $\begin{array}{l}\text { lower Napo/ } \\
\text { lower } \\
\text { Aguarico }\end{array}$ & QCAZ25386 & Ecuador & Orellana & La Selva Lodge & 9 & DQ353833 & \\
\hline Bolitoglossa & cf. peruviana & $\begin{array}{l}\text { lower Napo/ } \\
\text { lower } \\
\text { Aguarico }\end{array}$ & QCAZ25387 & Ecuador & Orellana & La Selva Lodge & 9 & DQ353836 & KC614450 \\
\hline Bolitoglossa & cf. peruviana & $\begin{array}{l}\text { lower Napo/ } \\
\text { lower } \\
\text { Aguarico }\end{array}$ & QCAZ25420 & Ecuador & Orellana & La Selva Lodge & 9 & DQ353838 & \\
\hline Bolitoglossa & cf. peruviana & $\begin{array}{l}\text { lower Napo/ } \\
\text { lower } \\
\text { Aguarico }\end{array}$ & QCAZ25421 & Ecuador & Orellana & La Selva Lodge & 9 & DQ353832 & \\
\hline Bolitoglossa & cf. peruviana & $\begin{array}{c}\text { lower Napo/ } \\
\text { lower } \\
\text { Aguarico }\end{array}$ & QCAZ25422 & Ecuador & Orellana & La Selva Lodge & 9 & DQ353834 & \\
\hline Bolitoglossa & cf. peruviana & $\begin{array}{c}\text { lower Napo/ } \\
\text { lower } \\
\text { Aguarico }\end{array}$ & QCAZ25425 & Ecuador & Orellana & La Selva Lodge & 9 & DQ353839 & \\
\hline
\end{tabular}


Table 1 Sample information (Continued)

\begin{tabular}{|c|c|c|c|c|c|c|c|c|c|}
\hline Bolitoglossa & cf. peruviana & $\begin{array}{l}\text { lower Napo/ } \\
\text { lower } \\
\text { Aguarico }\end{array}$ & QCAZ25426 & Ecuador & Orellana & La Selva Lodge & 9 & DQ353837 & \\
\hline Bolitoglossa & cf. peruviana & $\begin{array}{l}\text { lower Napo/ } \\
\text { lower } \\
\text { Aguarico }\end{array}$ & QCAZ25592 & Ecuador & Orellana & $\begin{array}{l}\text { Auca } 14 \text { Rd near } \\
\text { Coca }\end{array}$ & 6 & DQ353831 & KC614448 \\
\hline Bolitoglossa & cf. peruviana & $\begin{array}{l}\text { lower Napo/ } \\
\text { lower } \\
\text { Aguarico }\end{array}$ & QCAZ25593 & Ecuador & Orellana & $\begin{array}{l}\text { Auca } 14 \text { Rd near } \\
\text { Coca }\end{array}$ & 6 & DQ353819 & KC614444 \\
\hline Bolitoglossa & cf. peruviana & $\begin{array}{l}\text { lower Napo/ } \\
\text { lower } \\
\text { Aguarico }\end{array}$ & QCAZ28404 & Ecuador & Sucumbíos & $\begin{array}{l}\text { Monte Tour, } \\
\text { Cuyabeno }\end{array}$ & 5 & KC614429 & KC614454 \\
\hline Bolitoglossa & cf. peruviana & $\begin{array}{l}\text { upper } \\
\text { Aguarico }\end{array}$ & QCAZ25784 & Ecuador & Sucumbíos & Aguarico & 11 & DQ353813 & \\
\hline Bolitoglossa & cf. peruviana & $\begin{array}{c}\text { upper } \\
\text { Aguarico }\end{array}$ & QCAZ25793 & Ecuador & Sucumbíos & Aguarico & 11 & DQ353814 & \\
\hline Bolitoglossa & cf. peruviana & $\begin{array}{l}\text { upper } \\
\text { Aguarico }\end{array}$ & QCAZ25794 & Ecuador & Sucumbíos & Aguarico & 11 & DQ353815 & KC614443 \\
\hline Bolitoglossa & cf. peruviana & $\begin{array}{l}\text { upper } \\
\text { Aguarico }\end{array}$ & QCAZ25795 & Ecuador & Sucumbíos & Aguarico & 11 & DQ353812 & \\
\hline Bolitoglossa & cf. peruviana & upper Napo & KU217421 & Ecuador & Napo & $\begin{array}{l}\text { Estación Biológica } \\
\text { Jatun Sacha (EBJS) }\end{array}$ & 3 & AY526170 & \\
\hline Bolitoglossa & cf. peruviana & upper Napo & QCAZ25289 & Ecuador & Napo & $\begin{array}{l}\text { Estación Biológica } \\
\text { Jatun Sacha (EBJS- } \\
\text { Inner Vision Lodge) }\end{array}$ & 3 & DQ353826 & \\
\hline Bolitoglossa & cf. peruviana & upper Napo & QCAZ25294 & Ecuador & Napo & $\begin{array}{l}\text { Estación Biológica } \\
\text { Jatun Sacha (EBJS- } \\
\text { Inner Vision Lodge) }\end{array}$ & 3 & DQ353816 & \\
\hline Bolitoglossa & cf. peruviana & upper Napo & QCAZ25317 & Ecuador & Napo & Cando & 7 & DQ353822 & \\
\hline Bolitoglossa & cf. peruviana & upper Napo & QCAZ25318 & Ecuador & Napo & Cando & 7 & DQ353824 & \\
\hline Bolitoglossa & cf. peruviana & upper Napo & QCAZ25319 & Ecuador & Napo & Cando & 7 & DQ353823 & \\
\hline Bolitoglossa & cf. peruviana & upper Napo & QCAZ25320 & Ecuador & Napo & Cando & 7 & DQ353821 & KC614445 \\
\hline Bolitoglossa & cf. peruviana & upper Napo & QCAZ25355 & Ecuador & Pastaza & $\begin{array}{l}\text { Sta Clara (finca de } \\
\text { Tapia) }\end{array}$ & 8 & DQ353818 & \\
\hline Bolitoglossa & cf. peruviana & upper Napo & QCAZ25455 & Ecuador & Napo & $\begin{array}{l}\text { Estación Biológica } \\
\text { Jatun Sacha (EBJS) }\end{array}$ & 3 & DQ353829 & \\
\hline Bolitoglossa & cf. peruviana & upper Napo & QCAZ25747 & Ecuador & Napo & $\begin{array}{l}\text { Estación Biológica } \\
\text { Jatun Sacha (EBJS- } \\
\text { Inner Vision Lodge) }\end{array}$ & 3 & DQ353817 & \\
\hline Bolitoglossa & cf. peruviana & upper Napo & QCAZ25753 & Ecuador & Napo & $\begin{array}{l}\text { Estación Biológica } \\
\text { Jatun Sacha (EBJS- } \\
\text { Inner Vision Lodge) }\end{array}$ & 3 & DQ353827 & KC614446 \\
\hline Bolitoglossa & cf. peruviana & upper Napo & QCAZ25758 & Ecuador & Napo & $\begin{array}{l}\text { Estación Biológica } \\
\text { Jatun Sacha (EBJS- } \\
\text { Inner Vision Lodge) }\end{array}$ & 3 & DQ353825 & \\
\hline Bolitoglossa & cf. peruviana & upper Napo & QCAZ25771 & Ecuador & Napo & $\begin{array}{l}\text { Estación Biológica } \\
\text { Jatun Sacha (EBJS- } \\
\text { Inner Vision Lodge) }\end{array}$ & 3 & DQ353828 & \\
\hline Bolitoglossa & cf. peruviana & upper Napo & QCAZ25872 & Ecuador & Napo & $\begin{array}{l}\text { Cando (Serena } \\
\text { North side) }\end{array}$ & 7 & DQ353820 & \\
\hline Bolitoglossa & equatoriana & equatoriana & $\begin{array}{l}\text { QCAZ05930 } \\
(=\text { LSUMZ- } \\
\text { H12838) }\end{array}$ & Ecuador & Sucumbíos & Cuyabeno Reserve & 5 & $\begin{array}{l}\text { AY526169 Previously } \\
\text { reported as B. } \\
\text { peruviana (Parra-Olea et } \\
\text { al 2004) }\end{array}$ & \\
\hline Bolitoglossa & equatoriana & equatoriana & QCAZ28221 & Ecuador & Sucumbíos & $\begin{array}{l}\text { Pto Bolivar } \\
\text { (Cuyabeno) }\end{array}$ & 5 & KC614428 & \\
\hline
\end{tabular}


Table 1 Sample information (Continued)

\begin{tabular}{|c|c|c|c|c|c|c|c|c|c|}
\hline Bolitoglossa & equatoriana & equatoriana & QCAZ37304 & Ecuador & Orellana & Tiputini Reserve & 2 & DQ353845 & KC614452 \\
\hline Bolitoglossa & palmata & & KU217422 & Ecuador & Napo & $\begin{array}{l}\text { Guacamayos } 31 \text { km } \\
\text { de Baeza }\end{array}$ & 4 & AY526164 & \\
\hline Bolitoglossa & $\begin{array}{l}\text { paraensis } \\
\text { (Acre) }\end{array}$ & & $\begin{array}{l}\text { LSUMZH- } \\
13735\end{array}$ & Brazil & Acre & $\begin{array}{l}5 \mathrm{~km} \mathrm{~N} \text { Porto } \\
\text { Walter }\end{array}$ & - & AY526168 & \\
\hline Bolitoglossa & $\begin{array}{l}\text { paraensis } \\
\text { (Ituxi) }\end{array}$ & & $\begin{array}{l}\text { LSUMZH- } \\
3086\end{array}$ & Brazil & Amazonas & $\begin{array}{l}\text { Rio Ituxi at } \\
\text { Madeireira Scheffer }\end{array}$ & - & AY526167 & \\
\hline Bolitoglossa & $\begin{array}{l}\text { paraensis } \\
\text { (Jurua) }\end{array}$ & & 3098 & Brazil & Amazonas & Río Jurua INPA & - & AY526166 & \\
\hline Bolitoglossa & sima & & MVZ163575 & Colombia & & Valle de Cauca & - & AY526172 & \\
\hline Bolitoglossa & sp. (Chilma) & & QCAZ39981 & Ecuador & Carchi & Chilma Bajo & 16 & KC614431 & KC614456 \\
\hline Bolitoglossa & sp. (Condor) & & QCAZ41724 & Ecuador & $\begin{array}{l}\text { Zamora- } \\
\text { Chinchipe }\end{array}$ & $\begin{array}{l}\text { Cordillera del } \\
\text { Cóndor, near San } \\
\text { Miguel de Las } \\
\text { Orquídeas }\end{array}$ & 17 & KC614432 & KC614456 \\
\hline Bolitoglossa & $\begin{array}{l}\text { sp. } \\
\text { (ECSanFran) }\end{array}$ & & JCS 019 & Ecuador & $\begin{array}{l}\text { Zamora- } \\
\text { Chinchipe }\end{array}$ & $\begin{array}{l}\text { Estación Científica } \\
\text { San Francisco }\end{array}$ & 18 & KC699921 & KC699927 \\
\hline
\end{tabular}

Taxon, voucher specimen museum catalogue number, geographical locality (country, province/territory and locality), locality number from Figure 1, and GenBank accession numbers. Institutional abbreviations are as listed in [36] with the following additions: BU = Boston University (USA), DFCH-USFQ = Diego Francisco Cisneros-Heredia at Universidad San Francisco de Quito (Ecuador), FHGO = Fundación Herpetológica Gustavo Orcés (Ecuador), QCAZ = Museo de Zoología

Pontificia Universidad Católica (Ecuador), JCS = field collection Juan Carlos Sánchez.

specimen from northwestern Ecuador (B. sp. Chilma) is recovered as sister to the rest of our east Andean and Amazonian specimens ( $\mathrm{pp}=0.96$; Figure 3 ). Its relationship with the lowland Amazonian species B. paraensis (Acre) is undefined. Specimens from the high altitude eastern slopes of the southern Andes of Ecuador (B. sp. ECSanFran, B. sp. Condor, B. palmata; localities 4, 18, 19; 1800-2000 masl) are all, with varying resolution and including the extreme lowland Brazilian B. paraensis, sister to the upper Amazonian species B. peruviana and B. equatoriana.

\section{Nuclear phylogeny}

Phylogenetic analysis of Rag1 alone (19 specimens) reveals weak phylogeographic structure (Figure 5), perhaps due to incomplete lineage sorting and the longer coalescence times of slowly evolving nuclear genes. As in the mtDNA phylogeny, the high Andean specimen from northwestern Ecuador (B. sp. Chilma) ( $\mathrm{pp}=0.99)$ is sister to the rest of our east Andean and Amazonian specimens. The remaining internal relationships are less defined. The combined analysis (Rag1 and Cytb from 19 specimens; Additional file 5) is intermediate in resolution between the single gene analyses.

\section{Patterns of diversity in the upper Amazon}

Similar high diversity has been found previously in Amazonian frogs, with morphological, bioacoustic, and molecular data across ecologically and evolutionarily diverse clades, including the pattern of elevation and latitude in discerning upper Amazonian clades. Specifically, the geographical distribution of mtDNA clades in
Bolitoglossa is approximately coincident with those identified for the terrestrial leaf litter frog Pristimantis "ockendeni" [5] (now split into three species [37]) and Engystomops frogs [38]. The major phylogeographic divergence within northwestern Amazonian terrestrial frogs of the species Engystomops (=Physalaemus) petersi is also altitudinal in the Napo Basin [39-41], although elevation was not an important overall biogeographic signal at broader scales in that species [39]. Given the paucity of data on the phylogenetic diversity and finescale phylogeographic structure of upper Amazonian vertebrates in this region, the extent to which elevation has promoted speciation and maintains species identity (as has been inferred for salamanders [42-44] and other taxonomic groups in North [45] and Central America [46]) requires further and detailed analyses combining genetic, phenotypic, and geographical information.

\section{Bolitoglossa species richness and relevance for conservation}

The high divergence and pronounced geographic structuring among subclades of these salamanders support our view that additional species of Bolitoglossa should be recognized in the upper Amazon region of Ecuador; more species exist than were previously recognized based on existing morphological descriptions and field surveys $[18,23,35,47-50]$. We propose that at least six upper Amazonian species (four within B. peruviana sensu lato, two within B. equatoriana sensu lato) be recognized, rather than the previously designated two ( $B$. equatoriana and $B$. peruviana). Additionally, perhaps two more species should be named from higher 


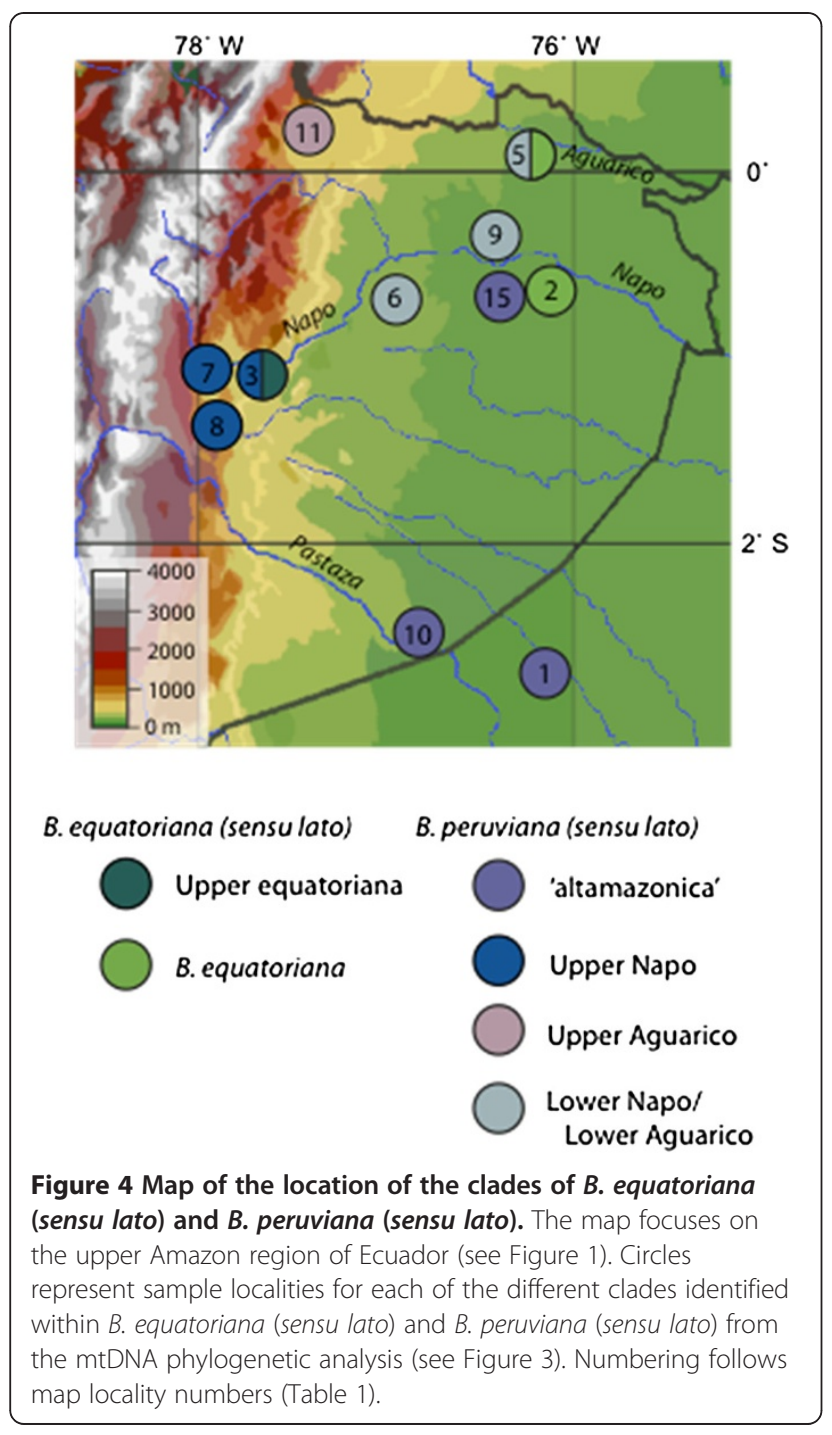

elevations to the south (B. sp. Condor and $B$. sp. ECSanFran). It is doubtful that any Ecuadorean salamander are conspecific with topotypic B. peruviana because the geographic distance from the most lowland Ecuadorean sample to the B. peruviana type locality is greater than the ranges of the three or four phylogenetic species (i.e. clades, as described here) within Ecuador. The situation with $B$. equatoriana is also difficult, although the geographic proximity of the $B$. equatoriana type locality to specimens of the "lower equatoriana" clade suggests that this group is B. equatoriana sensu stricto. From northern Ecuador, in the westward drainage of the Andes Mountains, we have a single exemplar of a currently unnamed highly divergent species ( $B$. sp. Chilma). Our study reveals that many taxonomic problems remain to be resolved for these morphologically similar species and work on this topic is currently underway (Wake et al. in prep.). Further alpha-taxonomic work and extensive sampling from the upper Amazon and Andes is much needed, though hampered by difficulty in collecting these rare amphibians from remote regions.

High genetic differentiation within "species" of Neotropical salamanders is not unusual in the few molecular studies on these to date (e.g. [51-54], Rovito et al. in prep). Garcia-Paris et al. [55] found genetic structuring at fine geographical scales for salamanders in continuous habitat in montane Costa Rica. In a study of allozyme variation, Hanken \& Wake [20] reported that South American Bolitoglossa displayed intra-site heterogeneity, high differentiation within species-groups, and diversity levels comparable to among species of different genera in North America. Studies of Neotropical frog taxa have also found mtDNA differentiation to be particularly high among populations, sometimes also suggestive of distinct species $[5,8,9,56,57]$, though not always [11]. The low vagility, high philopatry, small home ranges, and non-migratory life history of direct-developing salamanders and terrestrial frogs may promote high levels of local differentiation as a result of restricted gene flow [58], ultimately producing high speciation rates.

Our survey of salamanders from upper Amazon drainage of Ecuador indicates that upper Amazonian salamander species' distributions are much smaller than previously assumed. Accordingly, a revision of current conservation assessments may be required. The IUCN Red List considers all salamander species east of the Andes as data deficient with regard to their risk

Table 2 Average within- and among-clade Cytb K2P corrected distances for the Ecuadorean upper Amazon salamanders

\begin{tabular}{llcccccc}
\hline & Clade & $\mathbf{1}$ & $\mathbf{2}$ & $\mathbf{3}$ & $\mathbf{4}$ & $\mathbf{5}$ & $\mathbf{6}$ \\
\hline 1 & peruviana-lower Napo/lower Agua Rico & $\mathbf{0 . 0 3 3} \pm \mathbf{0 . 0 0 5}$ & \pm 0.013 & \pm 0.011 & \pm 0.013 & \pm 0.017 & \pm 0.014 \\
2 & peruviana-upper Napo & 0.119 & $\mathbf{0 . 0 2 1 \pm 0 . 0 0 4}$ & \pm 0.011 & \pm 0.012 & \pm 0.016 & \pm 0.017 \\
3 & peruviana-altamazonica & 0.110 & 0.108 & $\mathbf{0 . 0 6 2 \pm 0 . 0 0 8}$ & \pm 0.010 & \pm 0.017 & \pm 0.015 \\
4 & peruviana- Agua Rico & 0.111 & 0.095 & 0.095 & $\mathbf{0}$ & \pm 0.018 & \pm 0.017 \\
5 & upper equatoriana & 0.161 & 0.156 & 0.184 & 0.163 & $\mathbf{0}$ & \pm 0.014 \\
6 & equatoriana Brame \& Wake 1972 & 0.136 & 0.158 & 0.161 & 0.153 & 0.117 & $\mathbf{0 . 0 2 2} \pm \mathbf{0 . 0 0 5}$
\end{tabular}

Mean p-distances between subclades are written on the lower diagonal \pm standard error on the upper matrix; mean intraclade p-distances \pm standard error lie on the diagonal (bold type). 
Table 3 Average within- and among-clade Rag1 uncorrected p-distances for the Ecuadorean upper Amazon salamanders

\begin{tabular}{llcccccc}
\hline & Clade & $\mathbf{1}$ & $\mathbf{2}$ & $\mathbf{3}$ & $\mathbf{4}$ & $\mathbf{5}$ & $\mathbf{6}$ \\
\hline 1 & peruviana-lower Napo/lower Agua Rico & $\mathbf{0 . 0 1 3} \pm \mathbf{0 . 0 0 3}$ & \pm 0.003 & \pm 0.003 & \pm 0.005 & \pm 0.005 & \pm 0.003 \\
2 & peruviana-upper Napo & 0.012 & $\mathbf{0 . 0 0 6 \pm 0 . 0 0 3}$ & \pm 0.002 & \pm 0.006 & \pm 0.005 & \pm 0.004 \\
3 & peruviana-altamazonica & 0.015 & 0.011 & $\mathbf{0 . 0 1 6 \pm 0 . 0 0 3}$ & \pm 0.005 & \pm 0.005 & \pm 0.003 \\
4 & peruviana- Agua Rico & 0.025 & 0.027 & 0.028 & $\mathbf{n c}$ & \pm 0.007 & 0.006 \\
5 & upper equatoriana & 0.023 & 0.022 & 0.024 & 0.033 & $\mathbf{n c}$ & \pm 0.003 \\
6 & equatoriana Brame \& Wake 1972 & 0.015 & 0.013 & 0.016 & 0.26 & 0.008 & nc \\
\hline
\end{tabular}

Mean p-distances between subclades are written on the lower diagonal \pm standard error on the upper matrix; mean intraclade $\mathrm{p}$-distances \pm standard error lie on the diagonal (bold type).

assessment criteria (http://www.iucnredlist.org/). The Amazonian species are all thought to have very wide geographical distributions (e.g. B. altamazonica is purported to have a distribution including Colombia, Brazil, Venezuela, Ecuador, Peru, and Bolivia [12]) and therefore large population sizes are assumed; such widespread species have accordingly been considered of "Least Concern" [59-61]. Our findings suggest that wide geographical distributions are unlikely and species must be reassessed to take into account the possibility of smaller geographic distributions and concomitantly smaller population sizes, which might place these species at greater risk due to localized environmental degradation. Results to date imply that the salamander species richness in the Andes and upper Amazon has been substantially underestimated.

\section{Conclusions}

Minimal estimates for the timing of diversification of the South American salamanders of the genus Bolitoglossa, based on our studies of eastern Ecuadorean species, indicate that plethodontids colonized South America ca. 23 MYA. These findings are consistent with an Early Miocene, rather than Pliocene, initial closure of the Isthmus of Panama. Within South America, Bolitoglossa have considerable genetic diversity at nuclear and mitochondrial loci. There is a high cryptic diversity of distinct lineages, even within a relatively small geographic area of the Andes and upper Amazon of Ecuador. Our molecular phylogenetic findings suggest that salamander species richness in South America may be seriously underestimated, in large part because of extensive morphological similarity and lack of sampling. This implies that many more species of Bolitoglossa should be recognized in the upper Amazon and Andes.

\section{Methods}

\section{Taxon sampling}

Specimens were collected opportunistically from tropical forest localities in Ecuador (Figure 1). Individuals were euthanized using chloretone or MS-222 with approved protocols, liver tissue removed, then fixed with $10 \%$ formalin and stored in $70 \%$ ethanol. Tissue samples were stored in pure ethanol until DNA analysis. Voucher specimens are deposited at the Museo de Zoología, Pontificia Universidad Católica del Ecuador (QCAZ) and Fundación Herpetológica Gustavo Orcés collections (FHGO). Sample localities, museum voucher numbers, and Genbank accession numbers are listed in Table 1. For upper Amazonian localities, specimens were assigned to B. equatoriana Brame \& Wake, 1972 [35] or B. peruviana (Boulenger, 1883; see [18]) based on body size, shape and colouration.

\section{DNA extraction, amplification, and sequencing}

Total genomic DNA was extracted from ethanolpreserved liver tissue using a Qiagen DNEasy kit (Qiagen, Inc.) following the manufacturer's protocol, eluted in Buffer $\mathrm{AE}$, and stored at $-20^{\circ} \mathrm{C}$.

A 790 base pair fragment of the mitochondrial gene cytochrome $b(C y t b)$ was amplified by polymerase chain reaction (PCR) in an Applied Biosystems 2700 or 9700 thermocycler with parameters following [13]. PCR reactions used $0.3 \mu \mathrm{M}$ of each primer (MVZ15L and MVZ16H, [62]), PCR enhancing buffer (2.5 mM MgCl2, $10 \mathrm{mM}$ Tris $\mathrm{pH} 8.4,50 \mathrm{mM} \mathrm{KCl}, 0.02 \mathrm{mg}$ bovine serum albumin, $0.01 \%$ gelatin), $0.3 \mathrm{mM}$ of each dNTP, 0.625 units of taq DNA polymerase (Fermentas), and approximately 1 to 3 ng DNA per $50 \mu \mathrm{L}$ reaction. All amplifications included a negative control.

For representative individuals from major mitochondrial clades (see below), an 805 bp portion of the nuclear gene Rag1 was amplified using $0.2 \mu \mathrm{M}$ of each of the newly designed primers Rag1BolitoF (5'-CTT GAA CTA GGG GGC ATA CTC AGA AC-3') and Rag1BolitoR (5'-TGC CTG GCA TTC ATT TTC CGG AAA CG-3'). Typical PCR conditions were as follows: $5 \mu \mathrm{L}$ of $10 \mathrm{X}$ Promega PCR buffer, $1 \mathrm{mM} \mathrm{MgCl} 2,0.4 \mu \mathrm{M}$ of each dNTP, 0.5 to $1 \mu \mathrm{L}$ of a variety of taq polymerases, and approximately 2 to $4 \mathrm{ng}$ of DNA per $50 \mu \mathrm{L}$ PCR 


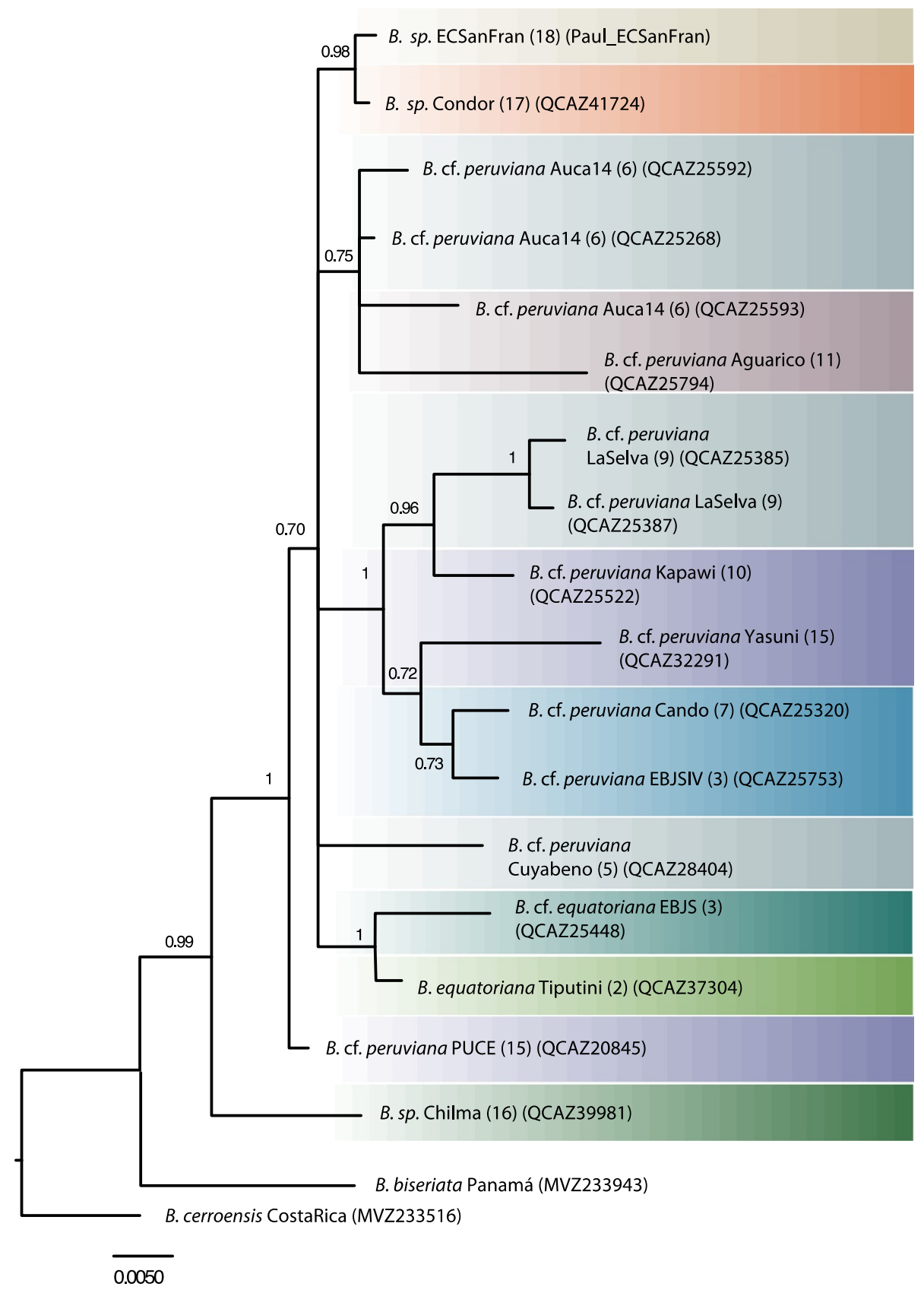

Figure 5 Rag1 phylogeny focusing on South American salamanders. The 50\% majority-rule phylogenetic tree of Rag1 inferred from Bayesian analyses. Posterior probabilities are located at nodes or above branches. Clades referred to in the text and derived from the combined analysis are noted in vertical. See Table 1 and Figure 1 for more information on sample localities.

reaction. Again all amplifications included a negative control.

PCR products of the correct molecular weight were excised after electrophoresis on an agarose gel and purified using Qiagen Gel Extraction kits (Qiagen, Inc.). Big Dye (Applied Biosystems Inc.) was used for cycle sequencing reactions, which were then sequenced on an Applied Biosystems 3730 capillary sequencer. All Cytb samples were sequenced in one direction using the MZV15L primer and a subset of five samples was sequenced in both directions. Detailed visual comparison of forward and reverse sequences in the overlapping regions showed no discordance in DNA sequence. All Rag1 samples were sequenced in both directions.

Bolitoglossa sequences were assembled and aligned in MacClade version 4.07 [63]. Nucleotide sequences were compared to protein sequences using BLASTX 
(http://www.ncbi.nlm.nih.gov/) to infer reading frames. Additional sequences were obtained from GenBank (Table 1).

\section{Divergence time estimates}

We estimated a Bolitoglossa species tree and divergence times via Bayesian methods using the "BEAST function in the program BEAST v. 1.6.2 [64]. The species tree was based on 805 bp of Rag1 for 31 taxa and 522 bp of Cytb for 110 taxa (Additional file 6). This allowed us to incorporate other previously published Cytb sequences for other South American Bolitoglossa and other major clades of Bolitoglossa from Central America [13]. The final data set included 60 "species" (tips), with representatives of the major lineages of $B$. equatoriana and $B$. peruviana, and two non-Bolitoglossa bolitoglossines (Pseudoeurycea and Thorius). We also included representatives of other plethodontids (Aneides, Ensatina, Plethodon, and Desmognathus) in order to take advantage of the crown group of plethodontids as a basal calibration point for our analyses. Missing data were filled in for taxa in which we only had one of the two genes.

This sequence data matrix was partitioned by gene and codon position using substitution and site heterogeneity models determined in Modeltest (Additional file 7, see Phylogenetic Analysis section). This analysis was based on an uncorrelated lognormal molecular clock and a Yule speciation prior across the tree. We calibrated the tree with a normally distributed prior basal calibration prior for the crown group Plethodontidae. A wide range of dates has been proposed for this node; from $~ 50$ to 99 MYA [21,65-70]. Therefore, we performed the analyses considering three alternative normally distributed calibrations priors for this node: 90 MYA (Std 6), 75 MYA (Std 6), and 60 (Std 6), which collectively span the entire range of age estimates for the node. We show the chronogram for the intermediate date (75 MYA), but we also report the ages of critical nodes in Additional file 2. The analysis was run twice independently for 20 million generations. The numerical results were visualized and compared using Tracer version 1.5 [71]. We estimated stationarity by examining the trace of likelihood values, which typically stabilized in less than one million generations, and we conservatively discarded 10\% (2 million generations) as burnin. In addition to the methods discussed above, we also analysed this divergence using a variety of other calibration strategies and priors and the results were highly consistent (i.e. deepest divergence within South American Bolitoglossa is always in the early to Middle Miocene). The purpose of this analysis was to test if divergence time estimates within an endemic South America clade of Bolitoglossa are significantly earlier than 3 MYA, which would suggest that Bolitoglossa either arrived in South American prior to the Pliocene Panamanian land bridge.
Ancestral areas of Bolitoglossa were reconstructed using the likelihood-based dispersal-extinction-cladogenesis analysis with LAGRANGE v. 12120508 [66,72]. The analyses were performed on the species tree chronogram of bolitoglossines based on the 75 MYA calibration. The geographical regions for the terminal taxa were either Central America, South America, or both regions, and we did not constrain dispersal probabilities.

\section{Phylogenetic analyses}

Modeltest version 3.7 [73] implemented with PAUP* [74] was used on each gene and codon position separately and both genes together to infer the best model of molecular evolution, which was selected using the Akaike Information Criterion. Sequence divergences between haplotypes and clades were calculated in MEGA4 [75] with a K2P correction [76] for Cytb and no correction for Rag1 (because of its lower mutation rate).

To reconstruct the evolutionary relationships among upper Amazonian and Andean Bolitoglossa with all sample and geographic information, Bayesian phylogenetic analyses were run in MrBayes version 3.1.2 [77] under a variety of partition and model settings for each codon position separately and some combined, as calculated from Modeltest (Additional file 7). Prior distributions on state frequencies, substitution rates, gamma distribution shape and the proportion of invariable sites were unlinked across all partitions and left at default values. Branch length prior was left at default for single gene analyses and was set to Unconstrained: Exponential (100) for the combined analysis. Site-specific rates were allowed to vary across partitions (ratepr $=$ variable). Temperature among the MCMC heated chains varied from 0.05 to 0.08 as needed to facilitate chain swapping. The starting tree was random as determined by MrBayes. Each analysis was run in two independent concurrent blocks of 3 million (Rag1) or 5 million (Cytb and combined) generations with sampling every 100 generations. The first 10000 trees (Rag1) or 15,000 trees (Cytb and combined) were discarded as burn-in. Convergence was assumed when chain swaps ranged between 0.4 and 0.8 , harmonic means were effectively identical from run 1 and run 2, and parameter PSRF values were approximately 1.00 . The partitioning, parameters and model chosen for the final tree were those that provided the highest Bayes factor compared to other models, except if the second best model differed in Bayes factor by less than 10 and was simpler [78]. Fifty percent majority consensus trees were built from the first two independent runs and visualized in FigTree version 1.3.1 (http://tree.bio. ed.ac.uk/). 


\section{Additional files}

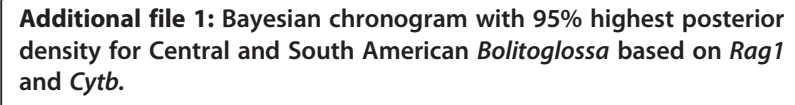

Additional file 2: Divergence time estimates based on alternative calibrations.

Additional file 3: Inter-individual K2P corrected sequence

divergence at cytochrome b. Sensu lato interspecific comparisons are shaded grey.

Additional file 4: Inter-individual uncorrected sequence divergence at Rag1. Sensu lato interspecific comparisons are shaded grey.

Additional file 5: Multilocus combined phylogeny.

Additional file 6: List of specimens (species, GenBank accession, continent) included in the divergence time analysis.

Additional file 7: Best likelihood models of sequence evolution for each codon position and combined positions.

\section{Competing interests}

The authors declare that they have no competing interests.

\section{Authors' contributions}

KRE carried out new specimen sampling, sequencing, and molecular genetic analyses, and drafted the manuscript. RMB did sequencing and divergence time and ancestral area analyses. DBW participated in study design, interpretation, and taxonomic issues. SCL participated in study design and interpretation. All authors contributed to writing the manuscript. All authors approved the final manuscript.

\section{Acknowledgements}

Specimens were collected with approval of the Ecuadorean Ministerio de Ambiente collection permits No. 006-IC-FAU-DBAP/MA and 016-IC-FAUDNBAP/MA. For samples or help with sample collection in the field we thank F. Ayala-Varela, T. Sugahara, S. Padilla, P. Menéndez, I.G. Tapia, D. CisnerosHeredia, C. Schneider, and F. Werner. For sequencing or assistance in the lab we thank N. Sharpe, D. Peiris, E. Bonosorcco, and J. Guayasamín. For logistical support in Ecuador we thank D. Duncan and turbodog, M. Bustamante, M. Guerra and D. Cannatella. S. O'Reilly provided computational resources. S. Mann created Figure 1. We thank L.A. Coloma for preliminary specimen identifications and comments on an earlier draft of the manuscript and S. Ron for use of photos. We thank J. Wiens, A. Crawford, and an anonymous reviewer for comments that improved the manuscript. This research was primarily funded by the Natural Sciences and Engineering Research Council (KRE and SCL), Queen's University Research funding (SCL), a Queen's University Field Research grant (KRE), and AmphibiaTree (NSF) (RMB and DBW). Some sequencing was also funded by "Inventario y caracterización Genética yMorfológica de la Diversidad de Anfibios, Reptiles y Aves de los Andes del Ecuador" code PIC08-0000470, funded by Secretaría Nacional de Educación Superior, Ciencia, Tecnología e Innovación de Ecuador, and issued to the Pontificia Universidad Católica del Ecuador.

\section{Author details}

'Department of Biology, Queen's University, Kingston, Ontario, Canada. ${ }^{2}$ Lehrstuhl für Zoologie und Evolutionsbiologie, Department of Biology, University of Konstanz, Universitätstrasse 10, Konstanz 78457, Germany. ${ }^{3}$ Current address: Institute of Biodiversity, Animal Health \& Comparative Medicine, College of Medical, Veterinary \& Life Sciences, University of Glasgow, Glasgow, UK. ${ }^{4}$ Department of Biological Science, University of Tulsa, Tulsa, Oklahoma, USA. ${ }^{5}$ Museum of Vertebrate Zoology and Department of Integrative Biology, University of California, Berkeley, California 94720-3160, USA.

Received: 7 June 2012 Accepted: 18 February 2013

Published: 4 March 2013

\section{References}

1. Wiens JJ: Global patterns of diversification and species richness in amphibians. Am Nat 2007, 170:A86-106.
2. Wiens JJ, Pyron RA, Moen DC: Phylogenetic origins of local-scale diversity patterns and causes of Amazonian megadiversity. Ecol Lett 2011, 14:643-652.

3. Carnaval AC, Hickerson MJ, Haddad CFB, Rodrigues MT, Moritz C: Stability predicts genetic diversity in the Brazilian Atlantic forest hotspot. Science 2009, 323:785-789.

4. Santos JC, Coloma LA, Summers K, Caldwell JP, Ree R, Cannatella DC: Amazonian amphibian diversity is primarily derived from late Miocene Andean lineages. PLoS Biol 2009, 7:e1000056.

5. Elmer KR, Dávila JA, Lougheed SC: Cryptic diversity and deep divergence in an upper Amazonian frog. Eleutherodactylus ockendeni. BMC Evol Biol 2007, 7:247.

6. Crawford AJ, Smith EN: Cenozoic biogeography and evolution in directdeveloping frogs of Central America (Leptodactylidae: Eleutherodactylus) as inferred from a phylogenetic analysis of nuclear and mitochondrial genes. Mol Phylogenet Evol 2005, 35:536-555.

7. Hedges SB, Duellman WE, Heinicke MP: New World direct-developing frogs (Anura: Terrarana): molecular phylogeny, classification, biogeography, and conservation. Zootaxa 2008, 1737:1-182.

8. Fouquet A, Gilles A, Vences M, Marty C, Blanc M, Gemmell NJ: Underestimation of species richness in neotropical frogs revealed by mtDNA analyses. PLoS One 2007, 10:e1109.

9. Fouquet A, Vences M, Salducci M-D, Meyer A, Marty C, Blanc M, Gilles A: Revealing cryptic diversity using molecular phylogenetics and phylogeography in frogs of the Scinax ruber and Rhinella margaritifer species groups. Mol Phylogenet Evol 2007, 43:567-582.

10. Schmid M, Steinlein C, Bogart JP, Feichtinger W, León P, La Marca E, Díaz LM, Sanz A, Chen S-H, Hedges SB: The chromosomes of Terraranan frogs. Cytogenet Genome Res 2010, 568:130-131.

11. Roberts JL, Brown JL, von May R, Arizabal W, Schulte R, Summers K: Genetic divergence and speciation in lowland and montane Peruvian poison frogs. Mol Phylogenet Evol 2006, 41:149-164.

12. AmphibiaWeb: Information on amphibian biology and conservation [web application]. http://amphibiaweb.org.

13. Parra-Olea G, García-París M, Wake DB: Molecular diversification of salamanders of the tropical American genus Bolitoglossa (Caudata: Plethodontidae) and its evolutionary and biogeographical implications. Biol J Linn Soc 2004, 81:325-346.

14. Wake DB: Diversity of Costa Rican salamanders. In Ecology and Evolution in the Tropics: A Herpetological Perspective. Edited by Donnelly MA, Crother BI, Guyer C, Wake MH, White ME. Chicago: University of Chicago Press; 2005:65-80

15. Kozak KH, Wiens JJ: Phylogeny, ecology, and the origins of climaterichness relationships. Ecology 2012, 93:S167-S181.

16. Farris DW, Jaramillo C, Bayona G, Restrepo-Moreno SA, Montes C, Cardona A, Mora A, Speakman RJ, Glascock MD, Valencia V: Fracturing of the Panamanian Isthmus during initial collision with South America. Geology 2011, 39:1007-1010.

17. Montes C, Cardona A, McFadden R, Morón SE, Silva CA, Restrepo-Moreno S, Ramírez DA, Hoyos N, Wilson J, Farris D, Bayona GA, Jaramillo CA, Valencia $\checkmark$, Bryan J, Flores JA: Evidence for middle Eocene and younger land emergence in Central Panama: Implications for Isthmus closure. Geol Soc Am Bull 2012, 124:780-799. online early.

18. Brame AH Jr, Wake DB: The salamanders of South America. Contr Sci Nat Hist Mus, Los Angeles County 1963, 69:1-73.

19. Dunn ER: The Salamanders of the Family Plethodontidae. Northampton, Massachusetts: Smith College; 1926.

20. Hanken J, Wake DB: Genetic differentiation among plethodontid salamanders (genus Bolitoglossa) in Central and South America: implications for the South American invasion. Herpetologica 1982, 38:272-287.

21. Wiens JJ, Parra-Olea G, García-París M, Wake DB: Phylogenetic history underlies elevational biodiversity patterns in tropical salamanders. Proc $R$ Soc Lond B 2007, 274:919-928.

22. Wake DB: What salamanders have taught us about evolution. Annu Rev Ecol Evol Syst 2009, 40:333-352.

23. Crump ML: Intrapopulation and interspecific variation of "standard" morphological characters of four closely related South American salamanders (Bolitoglossa), with description of habitat preferences. Herpetologica 1977, 33:415-426. 
24. Coates AG, Obando JA: The geologic evolution of the Central American Isthmus. In Evolution and Environment in Tropical America. Edited by Jackson JBC, Budd AF, Coates AG. Chicago: University of Chicago Press; 1996:21-56.

25. MacFadden BJ: Extinct mammalian biodiversity of the ancient New World tropics. Trends Ecol Evol 2006, 21:157-165.

26. Montes C, Bayona G, Cardona A, Buchs DM, Silva CA, Morón S, Hoyos N, Ramírez DA, Jaramillo CA, Valencia V: Arc-continent collision and orocline formation: closing of the central American seaway. J Geophys Res 2012, 117:B04105

27. Weir JT, Bermingham E, Schluter D: The Great American Biotic interchange in birds. Proc Natl Acad Sci USA 2009, 51:21737-21742.

28. Sedano RE, Burns KJ: Are the northern Andes a species pump for neotropical birds? Phylogenetics and biogeography of a clade of neotropical tanagers (Aves: Thraupini). J Biogeogr 2010, 37:325-343.

29. Weigt LA, Crawford AJ, Rand SA, Ryan MJ: Biogeography of the túngara frog, Physalaemus pustulosus: a molecular perspective. Mol Ecol 2005, 14:3851-3876

30. Pinto-Sánchez NR, Ibañez R, Madriñán S, Sanjur OI, Bermingham E, Crawford AJ: The Great American Biotic Interchange in frogs: multiple and early colonization of Central America by the South American genus Pristimantis. Mol Phylogenet Evol 2012, 62:954-972.

31. Cody S, Richardson JE, Rull V, Ellis C, Pennington RT: The great American biotic interchange revisited. Ecography 2010, 33:326-332.

32. Koepfli K-P, Gompper ME, Eizirik E, Ho C-C, Linden L, Maldonado JE, Wayne RK: Phylogeny of the Procyonidae (Mammalia: Carnivora): molecules, morphology and the Great American Interchange. Mol Phylogenet Evol 2007, 43:1076-1095.

33. Daza JM, Castoe TA, Parkinson CL: Using regional comparative phylogeographic data from snake lineages to infer historical processes in Middle America. Ecography 2010, 33:343-354.

34. Vanzolini PE, Heyer WR: The American herpetofauna and the Interchange. In The Great American Biotic Interchange. Edited by Stehli FG, Webb SD. New York and London: Plenum Press; 1985:475-487.

35. Brame AHJ, Wake DB: New species of salamanders (genus Bolitoglossa) from Colombia, Ecuador and Panama. Contr Sci Nat Hist Mus Los Angeles County 1972, 219:2-34

36. Leviton $A E$, Gibbs RH Jr: Standards in herpetology and ichthyology standard symbolic codes for institution resource collections in herpetology and ichthyology. Supplement No. 1: Additions and corrections. Copeia 1988, 1988:280-282.

37. Elmer KR, Cannatella DC: Three new species of leaflitter frogs from the upper Amazon forests: cryptic diversity within Pristimantis "ockendeni" (Anura: Strabomantidae) in Ecuador. Zootaxa 2008, 1784:11-38.

38. Funk WC, Caminer M, Ron SR: High levels of cryptic species diversity uncovered in Amazonian frogs. Proc R Soc Lond B 2012, 279:1806-1814

39. Funk WC, Caldwell JP, Peden CE, Padial JM, de la Riva I, Cannatella DC: Tests of biogeographic hypotheses for diversification in the Amazonian forest frog, Physalaemus petersi. Mol Phylogenet Evol 2007, 44:825-837.

40. Boul KE, Funk WC, Darst CR, Cannatella DC, Ryan MJ: Sexual selection drives speciation in an Amazonian frog. Proc R Soc Lond B 2007, 274:399-406.

41. Ron SR, Santos JC, Cannatella DC: Phylogeny of the tungara frog genus Engystomops (=Physalaemus pustulosus species group; Anura: Leptodactylidae). Mol Phylogenet Evol 2006, 39:392-403.

42. Kozak KH, Wiens JJ: Climatic zonation drives latitudinal variation in speciation mechanisms. Proc R Soc Lond B 2007, 274:2995-3003.

43. Kozak KH, Wiens JJ: Accelerated rates of climatic-niche evolution underlie rapid species diversification. Ecol Lett 2010, 13:178-1389.

44. Kozak KH, Weisrock DW, Larson A: Rapid lineage accumulation in a nonadaptive radiation: phylogenetic analysis of diversification rates in eastern North American woodland salamanders (Plethodontidae: Plethodon). Proc R Soc Lond B 2006, 273:539-546.

45. Badgley C: Tectonics, topography, and mammalian diversity. Ecography 2010, 33:220-231.

46. Smith SA, Nieto Montes de Oca A, Reeder TW, Wiens JJ: A phylogenetic perspective on elevational species richness patterns in Middle American treefrogs: why so few species in lowland tropical rainforests? Evolution 2007, 61:1188-1207.

47. Cisneros-Heredia DF: Amphibia, Caudata, Plethondontidae: Bolitoglossa equatoriana and Bolitoglossa biseriata: range extensions, new provincial records from Ecuador, and natural history. Check List 2006, 2:64-67.
48. Anfibios de Ecuador [en linea].http://zoologia.puce.edu.ec/Nertebrados/ anfibios/AnfibiosEcuador/InformacionPorFamilia.aspx.

49. Wake DB, Brame AH Jr: Notes on South American salamanders of the genus Bolitoglossa. Copeia 1966, No. 2:360-363.

50. Wake DB, Lynch JF: The distribution, ecology, and evolutionary history of plethodontid salamanders in tropical America. Sci Bull Nat Hist Mus Los Angeles County 1976, 25:1-65.

51. Larson A: A molecular phylogenetic perspective on the origins of a lowland tropical salamander family: I. Phylogenetic inferences from protein comparisons. Herpetologica 1983, 39:85-99.

52. García-París M, Wake DB: Molecular phylogenetic analysis of relationships of the tropical salamander genera Oedipina and Nototriton, with descriptions of a new genus and three new species. Copeia 2000, 2000:42-70.

53. García-París M, Parra-Olea G, Wake DB: Phylogenetic relationships within the lowland salamanders of the Bolitoglossa mexicana complex (Amphibia: Plethodontidae). In The Biology of Plethodontid Salamanders. Edited by Bruce RC, Jaeger RG, Houck LD. NY: Kluwer Academic/ Plenum Publishers; 2000:199-214

54. Parra-Olea G, Wake DB: Extreme morphological and ecological homoplasy in tropical salamanders. Proc Natl Acad Sci USA 2001, 98:788-7891.

55. García-París M, Good DA, Parra-Olea G, Wake DB: Biodiversity of Costa Rican salamanders: Implications of high levels of genetic differentiation and phylogeographic structure for species formation. Proc Natl Acad Sci USA 2000, 97:1640-1647.

56. Lougheed SC, Gascon C, Jones DA, Bogart JP, Boag PT: Ridges and rivers: A test of competing hypotheses of Amazonian diversification using a dartpoison frog (Epipedobates femoralis). Proc R Soc Lond B 1999, 266:1829-1835.

57. Crawford AJ: Huge populations and old species of Costa Rican and Panamanian dirt frogs inferred from mitochondrial and nuclear gene sequences. Mol Ecol 2003, 12:2525-2540.

58. Elmer KR, Dávila JA, Lougheed SC: Applying new inter-individual approaches to assess fine-scale population genetic diversity in a neotropical frog, Eleutherodactylus ockendeni. Heredity 2007, 99:506-515.

59. Azevedo-Ramos C, Reichle S, Almandáriz A, Castro F: Bolitoglossa altamazonica. In IUCN Red List of Threatened Species. 2006. Www.iucnredlist. org.

60. Coloma LA, Ron S, Cisneros-Heredia DF, Monteza Jl, Angulo A, Jungfer K-H: Bolitoglossa peruviana. In IUCN 2006. 2006 IUCN Red List of Threatened Species. www.iucnredlist.org.

61. Almandáriz A, Cisneros-Heredia DF, Jungfer K-H, Coloma LA, Ron S: Bolitoglossa equatoriana. In IUCN Red List of Threatened Species. 2006. www. iucnredlist.org.

62. Moritz C, Schneider CJ, Wake DB: Evolutionary relationships within the Ensatina eschscholtzii complex confirm the ring species interpretation. Syst Biol 1992, 41:273-291.

63. Maddison DR, Maddison WP: MacClade 4: Analysis of phylogeny and character evolution. Version 4.07. Sunderland: Sinauer Associates; 2003.

64. Drummond AJ, Rambaut A: BEAST: Bayesian evolutionary analysis by sampling trees. BMC Evol Biol 2007, 7:214

65. Mueller RL: Evolutionary rates, divergence dates, and the performance of mitochondrial genes in Bayesian phylogenetic analysis. Syst Biol 2006, 55:289-300

66. Ree RH, Moore BR, Webb CO, Donoghue MJ: A likelihood framework for inferring the evolution of geographic range on phylogenetic trees. Evolution 2005, 59:2299-2311.

67. Roelants K, Gower DJ, Wilkinson M, Loader SP, Biju SD, Guillaume K, Moriau $L$, Bossuyt F: Global patterns of diversification in the history of modern amphibians. Proc Natl Acad Sci USA 2007, 104:887-892.

68. Pyron RA, Wiens JJ: A large-scale phylogeny of Amphibia including over 2,800 species, and a revised classification of extant frogs, salamanders, and caecilians. Mol Phylogenet Evol 2011, 61:543-583.

69. Vieites DR, Min M-S, Wake DB: Rapid diversification and dispersal during periods of global warming by plethodontid salamanders. Proc Natl Acad Sci USA 2007, 104:19903-19907.

70. Zhang P, Wake DB: Higher-level salamander relationships and divergence dates inferred from complete mitochondrial genomes. Mol Phylogenet Evol 2009, 53:492-508

71. Rambaut A, Drummond AJ: Tracer version 1.4. http://beast.bio.ed.ac.uk/ Tracer. 
72. Ree RH, Smith SA: Maximum-likelihood inference of geographic range evolution by dispersal, local extinction, and cladogenesis. Syst Biol 2008, 57:4-14.

73. Posada D, Crandall K: MODELTEST: testing the model of DNA substitution. Bioinformatics 1998, 14:817-818.

74. Swofford DL: PAUP*. Phylogenetic Analysis Using Parsimony (*and Other Methods). version 4. Sunderland: Sinauer Associates; 2003.

75. Tamura K, Dudley J, Nei M, Kumar S: MEGA 4: Molecular evolutionary genetics analysis (MEGA) software version 4.0. Mol Biol Evol 2007, 24:1569-1599.

76. Kimura M: A simple method for estimating evolutionary rates of base substitutions through comparative studies of nucleotide sequences. J Mol Evol 1980, 16:111-120.

77. Huelsenbeck JP, Ronquist F: MRBAYES: Bayesian inference of phylogenetic trees. Bioinformatics 2001, 17:754-755.

78. Brown JM, Lemmon AR: The importance of data partitioning and the utility of Bayes Factors in Bayesian phylogenetics. Syst Biol 2007, 56:643-655

doi:10.1186/1471-2148-13-59

Cite this article as: Elmer et al:: Early Miocene origin and cryptic

diversification of South American salamanders. BMC Evolutionary Biology 2013 13:59.

\section{Submit your next manuscript to BioMed Central and take full advantage of:}

- Convenient online submission

- Thorough peer review

- No space constraints or color figure charges

- Immediate publication on acceptance

- Inclusion in PubMed, CAS, Scopus and Google Scholar

- Research which is freely available for redistribution 